\title{
Routine intraoperative cholangiography during laparoscopic cholecystectomy
}

\author{
Lacitignola Sebastiano*, Massafra Roberto and Manzo Fabio \\ Department of General Surgery, Clinica Bernardini, Taranto, Italy
}

\begin{abstract}
Objectives: The present study analyses the value of intraoperative cholangiography (IOC) performed in all patients during 22 years of surgical activity in three different Institutions and, as a result a continuous scientific concern on the matter, we developed a set of criteria that are analysed and discussed.

Materials and methods: We studied the patients subjected to laparoscopic cholecystectomy in three hospitals between January 1995 and December 2017. A group of patients were analysed, IOC was performed in all cases.

Results: we had a positive results of common bile duct stones (CBDS) unsuspected in more than 10\%, a finding that changed surgical management of patient after IOC. This procedure required a median period of 10 minutes and there were no complications caused by IOC.
\end{abstract}

Conclusions: Intraoperative cholangiography performed routinely represents an important tool in diagnosing unsuspected Common Bile Duct Stones (CBDS) during LC. This attitude has prevented further surgical treatment for all patients with asymptomatic biliary calculosis.

\section{Introduction}

The asymptomatic stones of the common bile duct (CBD) represents, even today, a considerable challenge for the surgeon despite the progress made both in endoscopy and laparoscopy. Just over 10 years ago, $10 \%$ of patients with symptomatic calculosis and $15 \%$ of those with acute cholecystitis had stones in the common bile duct. With the laparoscopic procedure the patients had the advantage of a more immediate and faster approach to cholecystectomy with a consequent reduction of choledocholithiasis. Even when all procedures are feasible, there are some techniques preferred to others. If the patient had cholecystectomy it is unanimous opinion that the choledochal lithiasis must be treated endoscopically through endoscopic retrograde cholangiopancreatography (ERCP) with Endoscopic Sphincterotomy (ES) reserving surgery in case of failure (5-10\%) [1]. If the gallbladder is "in situ" the treatment is controversial i.e. ERCP followed by laparoscopic cholecystectomy (LC). Another possibility is to perform the cholecystectomy and to remove the CBD stones through the laparoscopy or to abandon the stones with subsequent ERCP after LC. Many surgeons agree with these options in relation to their personal experience as well as the various protocols to be followed. Our personal tendency is to treat the CBDS in a single time using all the possible surgical strategies in our possession and this our attitude is also confirmed by the positive results obtained over the years.

\section{Materials and methods}

From January 1995 to December 2017 we were collected the data of 8918 patients undergoing LC for symptomatic lithiasis in three different Institutions. Routine Intraoperative Cholangiography (IOC) was successfully performed in 8806 patients $(98.8 \%)$. In the remaining 112 patients $(1.2 \%)$ the failure was due to the technical difficulties. The preoperative suspicion of CBDS was based on the patient's clinical history, on the possible appearance of jaundice or pancreatitis considering the high blood values of bilirubin $(>1.0 \mathrm{mg} / \mathrm{dl})$, alkaline phosphate $(>147 \mathrm{U} / \mathrm{dl})$, amylase $(>115 \mathrm{U} / \mathrm{dl})$ or the ultrasound documentation of CBDS or his diameter greater than $7 \mathrm{~mm}$. If none of these elements is present, we consider the IOC routine. The common bile stones were found in 1631 patients $(18,5 \%)$ with a preoperative diagnosis of 1458 cases $(89,3 \%)$ and 173 cases (10,6\%) discovered during the LC due to perioperative cholangiography. The ERCP with ES was performed successfully in 1221 patients $(83,7 \%)$ who had a preoperative diagnosis of CBDS. The remaining 237 patients $(16,2 \%)$ added to those they had an intraoperative diagnosis of CBDS were treated surgically as shown in table 1 . Operative cholangiography was attempted in all patients using a 4-Fr catheter and a choledochoscopy was performed using a 7-FR size fiberscope introduced through the cystic duct or choledochotomy to confirm the complete bile duct clearance. The patients had follow-up at 6-12-24 months and was not found a residual or recurrence stones. The postoperative complications reported two cases of bilioma after removal of the T-tube and these patients were treated with conservative therapy. No mortality was found.

\section{Discussion}

Biliary tract stones are the most-common cause of abdominal pain and the most-common complication of gallstones. Biliary colic or biliary tract disease is reportedly found in approximately $7 \%$ to $15 \%$ of adult patients presenting with abdominal pain [2]. The incidence of gallstones increases with age, with a prevalence of $30 \%$ by the age of 70 [3]. Approximately $80 \%$ of gallstones have silent presentation and the

${ }^{*}$ Correspondence to: Lacitignola Sebastiano, Contrada Sicarico 181/A, 70043 Monopoli , Italy, Tel: +39-330-840630; E-mail: lacitignola@libero.it

Received: November 02, 2019; Accepted: November 24, 2019; Published: November 28, 2019 
Table 1. Demographic table of patients undergoing laparoscopic cholecystectomy with IOC $(1995-2018)$

\begin{tabular}{|l|l|l|l|}
\hline $\begin{array}{l}\text { Total operated } \\
\text { patients }(8918)\end{array}$ & Age Range (14 - 92) & Men $3711(41.7 \%)$ & $\begin{array}{l}\text { Women 5207 } \\
(58.3 \%)\end{array}$ \\
\hline $\begin{array}{l}\text { IOC perfomed } 8806 \\
(98.8 \%)\end{array}$ & Failure 112 (1.2\%) & & \\
\hline Choledochal lithiasis & $1631(18.5 \%)$ & & \\
\hline Pre op diagnosis & $1458(89.3 \%)$ & & \\
\hline Intra op diagnosis & $173(10.6 \%)$ & & \\
\hline
\end{tabular}

most of patients remain symptoms free for decades. From $8 \%$ to $10 \%$ of individuals with asymptomatic gallstones develop symptoms within 5 years and only $5 \%$ requiring surgery [4]. From these data it's easy to understand that the treatment of gallbladder and bile duct can be variable especially when the diagnosis is made during the LC.

Therefore, we believe that the cholecystectomy cannot be considered complete if some stones are left in the bile duct. For this reason, we opt for a routine IOC that in our experience has allowed the diagnosis of CBDS for 295 patients for whom further endoscopic or surgical operation would be necessary. Indeed, the prevalence of asymptomatic CBDS ranges from 5,2\% to $12 \%$ [5] and it has been reported that more than half of patients with retained asymptomatic duct stones develop symptoms over time with $25 \%$ with serious complications [6].

Once the stones are diagnosed in the biliary duct during LC we are faced with a dilemma, in other words to remove the stones laparoscopically, to convert in open surgery, to abandon the stones waiting for these to escape from the choledochus spontaneously before performing the ERCP with ES subsequently or to make laparoendoscopic " rendezvous " at the same time. These different approaches are based not only on the ability of the surgeon and the endoscopist but also on the available instruments. All of this affects a different percentage of success, morbidity and mortality [7].

In the era of open cholecystectomy, choledocholithiasis was diagnosed at per-operative cholangiography and calculi were removed by CBDE like a natural extension of the operative procedure. Despite LC is considered an optimal approach with postoperative morbidity and mortality rates and duration of hospital stay lower than for standard cholecystectomy [8] "this new way to do surgery" has altered the approach to choledocholithiasis, as laparoscopic exploration of the bile duct, particularly for asymptomatic stones.

If on the one hand it's understandable that he whom performs 100 laparoscopic cholecystectomies or less per annum would be unlikely to develop or maintain the skills necessary for laparoscopic common bile duct exploration, on the other hand we believe that the CBDS must be treated at the same time of cholecystectomy both of costs and for effectiveness and safety. Even if the practice of routine operative cholangiography has been abandoned by many surgeons without valid reasons, its advantages are undeniable.

Indeed only few randomized trials are available comparing preoperative ERCP followed by LC with single stage LC and LCBDE $[9,10]$ and the same doubt continuous to exist on the method of exploration the biliary duct transcystic (TC) vs transcholedochal (TD) [11] and for closure of choledochotomy (T-tube vs biliary stent vs primary closure) [12]. From our positive results we state that the routine use of IOC at first does not alter the surgical times and this has been documented by other authors [13]. Secondly this management has allowed the occasional diagnosis of choledocal stones with a percentage value higher than reported in literature.

Another great advantage we obtained with the systemic use of the T-tube to close the common bile duct because this did not result in fistulae or biliary stricture or other complications in follow-up excepted for two biliomas treated conservatively. Moreover, the presence of the T-tube allowed to perform a cholangiography in order to control the clearance of biliary ducts. Many surgeons do not share this attitude and they prefer the primary closure or biliary stent of common bile duct, but the complications reported in literature [14] never convinced us to adopt these techniques. A Cocrhane systematic review by Martin [15] concluded that a single-stage treatment in the form of LCBDE via TC approach is the procedure of choice for intraoperatively discovered CBD calculi. Another option for LCBDE is his failure, therefore it is prudent to convert to open surgery.

\section{Conclusion}

Surgical agreement has not yet arrived at any consensus for adequate treatment of choledocholithiasis and till today the sequential treatment in the form of pre-operative ERCP followed by LC is considered as optimal therapy. Uncertainly has always existed for the treatment of underestimated CBS. The long learning curve of laparoscopic procedure has led us to prefer the LCBDE routinely and to solve in one stage management of cholelithiasis with choledocholitiasis.

\section{References}

1. Petelin J (1991) Laparoscopic approach to common duct pathology. Surg Lap Endos 1: 33-41. [Crossref]

2. Caddy GR, Tham TC (2006) Gallstone disease. Symptoms, diagnosis and endoscopic management of common bile duct stones. Best Pract Res Clin Gastroenterol 20: 10851101. [Crossref]

3. Kelly K, Weber S (2006) Cholecystitis. In: Jamagin WR, Belghiti J, Buchler MW et al., eds. Surgery of the liver, Biliary Tract and Pancreas 487-493.

4. Halldestam I, Enell EL, Kullman E, Borch K (2004) Development of symptoms and complications in individuals with asymptomatic gallstones. Br J Surg 91: 734-738. [Crossref]

5. Rosseland AR, Glomsaker TB (2000) Asymptomatic common bile duct stones. Eur J Gastroenterol Hepatol 12: 1171-1173. [Crossref]

6. Johnson AG, Hosking SW (1987) Appraisal of the management of bile duct stones. $\mathrm{Br}$ J Surg 74: 555-560. [Crossref]

7. Lacitignola S, Minardi M (2008) Management of common bile duct stones: a ten-years' experience at a tertiary care center. JSLS 12: 62-65. [Crossref]

8. Amaral PC, Azaro Filho EM, Galvão-Neto MP, Fortes MF, Souza EL, et al. (1988) Video laparo-cholecystectomy: casuistry of 1000 cases. J Soc Laparoendosc Surg 2: 141-145. [Crossref]

9. Rogers SJ, Cello JP, Horn JK, Siperstein AE, Schecter WP, et al. (2010) Prospective randomized trial of $\mathrm{LC}+\mathrm{LCBDE}$ vs $\mathrm{ERCP}+\mathrm{LC}$ for common bile duct stone disease Arch Surg 145: 28-33. [Crossref]

10. Cuschieri A, Lezoche E, Morino M, Croce E, Lacy A, et al. (1999) E.A.E.S. multicentre prospective randomized trial comparing two-stage vs single-stage management of patients with gallstone disease and ductal calculi. Surg Endosc 13: 952-957. [Crossref]

11. Honjun H, Yong J, Baoqiang W (2015) Laparoscopic common bile duct exploration choledochotomy versus trascystic approach? Surg Laparosc Endosc Percutan Tech 25: 218-222. [Crossref]

12. Yi HJ, Hong J, Min SH, Lee HK (2015) Long-term outcome of primary closure after laparoscopic common bile duct exploration combined with choledochoscopy. Surg Laparosc Endosc Percutan Tech 25: 250-253. [Crossref]

13. Vindal A, Chander J, Lal P, Mahendra B (2015) Comparison between intraoperative cholangiography and choledochoscopy for ductal clearance in laparoscopic CBD exploration: a prospective randomized study. Surg Endosc 29: 1030-1038. [Crossref]

14. Abellan Marcillo I, Qurashi K, Abrisqueta Carrion J, Martinez Isla A (2014) Laparoscopic common bile duct exploration. Lessons learned after 200 cases. Cir Esp 92: 341-347. [Crossref]

Copyright: (C2019 Sebastiano L. This is an open-access article distributed under the terms of the Creative Commons Attribution License, which permits unrestricted use, distribution, and reproduction in any medium, provided the original author and source are credited. 